\title{
LETTER-TO-EDITOR
}

\section{Safety and Cost Efficiency in Thyroid Surgery}

\author{
${ }^{1}$ Sabaretnam Mayilvaganan, ${ }^{2}$ Amit Agarwal \\ ${ }^{1}$ Assistant Professor, ${ }^{2}$ Professor \\ 1,2Department of Endocrine Surgery, Sanjay Gandhi Post Graduate Institute of Medical Sciences, Lucknow, Uttar Pradesh, India
}

Corresponding Author: Sabaretnam Mayilvaganan, Assistant Professor, Department of Endocrine Surgery, Sanjay Gandhi Post Graduate Institute of Medical Sciences, Lucknow, Uttar Pradesh, India, Phone: 9655851510, e-mail: drretnam@gmail.com

Dear editor,

We read with interest the article 'Safety and cost efficiency in thyroid surgery' by Young-Chul OT and Gough I. ${ }^{1}$ We congratulate the authors on their work which is aimed toward reducing the cost and also providing a safe approach with acceptable clinical indicators for the outcome of thyroid surgery. This is more so relevant in the developing world, where the endocrine surgeon has to balance between the usage of newer technology as well as curtailing the cost of the procedure. Sutureless thyroidectomy using vessel sealing devices which have become a routine in most centers. ${ }^{2}$

We have few queries which shall be of benefit for future readers. Although, clips achieve reliable vessel sealing, they have risk of dislodgment with tissue manipulation. The clip application also requires precise dissection of vessels prior to application and also believed to result in dense adhesions postsurgery, which shall make reoperation really difficult. ${ }^{3}$ We would like to have author's view regarding these issues. In highly vascular malignant and hyperthyroid tumors, surgeons may end up applying a lot of clips whereas the ultrasonic coagulating shears provide adequate vessel sealing and also saves time.

Regarding intraoperative nerve monitoring, we do agree with the authors that this procedure requires expertise and the operating team of surgeons and the anesthetist should be adequately trained to achieve optimum results. We agree with the authors that the factors, like extensive adhesions, difficult access and severe thyroiditis, determine the difficulty. However, in thyroid surgery, other intraoperative factors, like increased vascularity, inflammation, friability, fibrosis, large gland size and the grade of tubercle of Zuckerkandl, course of the recurrent laryngeal nerve, the position of the parathyroids, also determine the duration as well as the difficulty of surgery. ${ }^{4}$ Were these factors considered?

\section{REFERENCES}

1. Young-Chul OT, Gough I. Safety and cost efficiency in thyroid surgery. World J Endoc Surg 2014;6(3):101-106.

2. Chang LY, O'Neill C, Suliburk J, Sidhu S, Delbridge L, Sywak M. Sutureless total thyroidectomy: a safe and cost-effective alternative. ANZ J Surg 2011 Jul-Aug;81(7-8):510-514.

3. Harold KL, Pollinger H, Matthews BD, Kercher KW, Sing RF, Heniford BT. Comparison of ultrasonic energy, bipolar thermal energy, and vascular clips for the hemostasis of small-, medium-, and large-sized arteries. Surg Endosc 2003 Aug;17(8):1228-1230.

4. Schneider DF, Mazeh H, Oltmann SC, Chen H, Sippel RS. Novel thyroidectomy difficulty scale correlates with operative times. World J Surg 2014 Aug;38(8):1984-1989.

\section{Authors' Reply}

\author{
${ }^{1}$ Ian Gough, ${ }^{2}$ Oh Thomas Young-Chul \\ ${ }^{1}$ Professor, ${ }^{2}$ Former Fellow \\ ${ }^{1}$ Department of Surgery, University of Queensland, Brisbane, Australia \\ ${ }^{2}$ Department of Breast and Endocrine Surgery, Royal Brisbane and Women's Hospital, Brisbane, Australia
}

Corresponding Author: lan Gough, Wesley Medical Centre, 40 Chasely Street, Auchenflower-4066, Australia, e-mail: goughmed@ bigpond.com

Dear editor,

We thank Dr Sabaretnam and Dr Agarwal for their interest and comments on our article. ${ }^{1}$

We are pleased that they appreciated the objective of the study to show that safe and efficient thyroid surgery could be performed at relatively low cost with widely available simple equipment. 
Our practice is to perform total thyroidectomy or complete hemithyroidectomy without disturbance of the contralateral side so reoperative experience on our own cases is relatively limited. However, we have not encountered a particular problem with adhesions related to previously inserted titanium clips.

In our series, the patients were consecutive and unselected. The series was large enough to allow multivariate analysis of factors that affected the time of surgery. There was increased difficulty and increased operating time in patients under age 50, male gender, Graves' disease, large gland size and concurrent neck lymph node dissection. Nevertheless, the operation times for all patients were approximately 10 minutes faster than the times of combined series reporting the use of vessel sealing devices (VSDs). There do not appear to be series using VSDs reporting shorter operating times or lower complication rates than our experience using Ligaclips.

Regarding intraoperative nerve monitoring (IONM), in addition to the meta-analysis that showed no benefit of $\mathrm{IONM}_{,}{ }^{2}$ there has been more recent reporting of large multicenter experience again showing no benefit of IONM. ${ }^{3}$ This remains a subject of debate. ${ }^{4}$ The authors' opinion are that IONM should not be considered routine surgical practice and certainly not 'standard of care'. We believe that careful operative technique is the best way to avoid recurrent laryngeal nerve injury in thyroid surgery. ${ }^{5}$

We estimated that the time saved by using an automatic titanium Ligaclip applier was 5 to 10 minutes. If we had used a single clip applier or sutures and diathermy alone, the operative times would still have compared favorably to times reported for VSDs and at even lower cost.

\section{REFERENCES}

1. Oh TY-C, Gough IR. Safety and cost efficiency in thyroid surgery. World J Endo Surg 2014;6(3):101-106.

2. Higgins TS, Gupta R, Ketcham AS, et al. Recurrent laryngeal nerve monitoring versus identification alone on postoperative true vocal cord palsy: a meta-analysis. Laryngoscope 2011;121(5):1009-1017.

3. Chung TK, Rosenthal EL, Porterfield JR, et al. Examining national outcomes after thyroidectomy with nerve monitoring. J Am Coll Surg 2014;219(4):765-770.

4. Delbridge L, Gough I, Lisewski D, et al. Consensus statements in surgery: intraoperative nerve monitoring in thyroid surgery. ANZJ Surg 2015;85(1-2):5-7.

5. Gough IR. Avoiding recurrent laryngeal nerve injury in thyroid surgery. ANZJ Surg 2014;84(11):895. 
